\title{
PERSONALIZED FEEDBACKS BASED ON LEARNING ANALYTICS TO ENHANCE THE LEARNING OF PROGRAMMING
}

\author{
Zahi Hodeib and Yvan Peter \\ Université de Lille, CNRS, UMR 9189, CRIStAL, F-59000 Lille, France
}

\begin{abstract}
This paper presents a new learning environment called Codeclick. The purpose of the environment is to provide personalized feedbacks and recommendations to novices in order to improve their learning of programming. Codeclick uses a Learning Analytics statistical approach based on the learners' digital traces. It also provides visualizations to allow learners to monitor their performance levels and identify their errors. These visualizations also allow the teacher to identify blocking problems and intervene at the appropriate moment. Preliminary results of the design and the use of Codeclick show an improvement in the learners' performance, in terms of the number of errors made, as well as the time spent per activity.
\end{abstract}

\section{KEYWORDS}

Programming, Learning Analytics, Feedbacks, Visualizations, Higher Education

\section{INTRODUCTION}

Recently, programming courses has become essential in the curriculum of several academic fields. However, their practical learning represents a difficulty for the majority of students, and particularly for novices (Lahtinen et al., 2005). This explains the high failure and dropout rates in introductory to programming courses (Bennedsen and Caspersen, 2007). Among the main reasons, we can mention the large number of learners who are enrolled to these practical activities and the differences in terms of pedagogical capacities and skills between them, which considerably increases the workload of the teachers to ensure a good follow-up (Gulwani et al., 2014). Consequently, these teachers will be unable to support learners by providing personalized feedbacks and recommendations, during these practical activities. However, several studies consider that providing an appropriate feedback to learners at the right moment could be among the best contributors to improving the learning process (Hattie and Timperley, 2007).

On the other hand, several programming learning platforms have been developed in recent years. Some of these educational systems provide the learners with practical programming activities supported by certain forms of feedbacks. However, the quality of the feedbacks delivered by the majority of these systems remains unsatisfactory for the learners (Ferguson, 2011). Actually, these feedbacks suffer from a poor relevance, since they are based on static information about the learning process. Consequently, these feedbacks do not clearly reflect the performance level really achieved by the learners. In addition, they are mainly focused on displaying the errors committed by the learner, in the manner of traditional compilers, whose messages are incomprehensible and do not provide solutions on how to overcome these problems (Keuning et al., 2019). Moreover, the huge amount of information, also called learners' digital traces, resulting from the interaction of the latter with the educational platforms, paved the way for the development of the Learning Analytics (LA) field. LA mainly aims to effectively use these digital traces to improve the learning experience (Pardo et al., 2015). The following section presents an overview of the main works carried out to exploit the learners' digital traces with the aim to support practical learning of programming. 


\section{RELATED WORK}

According to the first international conference on Learning Analytics and Knowledge (LAK11), LA was defined as the measurement, collection, analysis and reporting of data about learners and their contexts, for purposes of understanding and optimizing learning and the environment in which it occurs (Siemens and Long, 2011). In other words, LA aims to use the learners' digital traces to provide teachers and institutions with capabilities allowing them to follow the progress of their students, identify the main causes of failure, intervene with personalized feedbacks and making decisions to adapt their teaching methods (Villamañe et al., 2018). Referring to the literature, the main approaches used by LA, namely, prediction, visualizations via Learning Analytics Dashboards (LAD) and statistical techniques, to assess learners' performance and consequently provide feedbacks and recommendations to assist them (Nunn et al., 2016). Several Intelligent Tutoring Systems (ITS), Intelligent Laboratories, tools and web applications have been developed to assist learners or teachers. Table 1 presents and compares some of these technologies, by focusing mainly on the types of feedbacks and recommendations delivered and the kind of data collected and used to generate them.

Table 1. Works offering feedbacks based on learners' digital traces

\begin{tabular}{|c|c|c|c|}
\hline Technologies & Publications & $\begin{array}{l}\text { Feedbacks and } \\
\text { Recommendations }\end{array}$ & Digital Traces \\
\hline \multirow[t]{2}{*}{ Web Applications } & $\begin{array}{l}\text { WebToTeach (Arnow and } \\
\text { Barshay, 1999) }\end{array}$ & Alerts and Messages & Code \\
\hline & Data2U (Khan and Pardo, 2016) & Dashboards & Interactions \\
\hline \multirow[t]{3}{*}{ Tools } & Retina (Murphy et al., 2009) & Recommendations Reports & Time and Errors \\
\hline & Ontask (Pardo et al., 2018) & Personalized Messages & Interactions \\
\hline & $\begin{array}{l}\text { Course Signal (Arnold and Pistilli, } \\
\text { 2012) }\end{array}$ & Personalized Emails & $\begin{array}{l}\text { Histories, Grades, } \\
\text { Interactions }\end{array}$ \\
\hline \multirow{2}{*}{$\begin{array}{l}\text { Intelligent } \\
\text { Laboratories }\end{array}$} & Smart Lab (Alammary et al., 2012) & Personalized Visualizations & Interactions \\
\hline & Lab4CE (Broisin et al., 2017) & Messages, Visualizations & Code, Interactions \\
\hline
\end{tabular}

Generally, these systems collect different information and traces about the learners (Code, Interactions, Number of clicks, Screenshots and Time spent per task). However, numerous of these systems provide inadequate feedbacks and do not take into account the real and personalized needs of each learner, especially their current profile and knowledge. Therefore, the learner finds himself overwhelmed by the enormous amount of inconsistent and incomprehensible feedbacks received and remains blocked in his activity. Moreover, some researchers have noticed that many learners cannot even understand certain feedbacks, or even the results provided by the LAD, therefore these latter will have no impact on the learners' performance. Rather, they could have negative psychological effects on some of them (Corrin and de Barba, 2015). In this paper, we present a new learning environment called Codeclick, relying on a LA approach, to allow novices to practice programming, supported by personalized feedbacks and recommendations, but also visualizations illustrating their performance levels and errors.

\section{CONTRIBUTION}

Codeclick is a programming learning environment offering practical activities intended for novices and their teachers. It was developed on the WAMP server using PHP with JavaScript and uses a MySQL database. Codeclick allows the learners to practice programming activities in two modes: Feedback mode (F) or Without Feedback mode (WF). The F mode provides personalized feedback to learner on code errors and recommendations on how to overcome them. Codeclick collects and analyzes the learners' digital traces (Codes, Number of clicks, Time spent by activity), classifies and stores them in a database. These digital traces are then used as indicators within a statistical analysis phase, to calculate the performance of each learner according to equation (1) and consequently generate feedbacks and visualizations illustrating this level of performance and the problems encountered during these activities. These visualizations allow the learner to understand the reasons of his weakness and compare himself to his colleagues. Also, they allow the 
teacher to identify the main causes of errors and the level of performance of each learner, which allows him to intervene and support the latter at the appropriate moment.

$$
\begin{array}{ll}
\mathrm{P}=100-2.5 * N C-3 * N E-T & \text { NC: Number of clicks } \\
& \text { NE: Number of Errors } \\
& \text { T: Time in minutes/Activity }
\end{array}
$$

In this equation, the performance of each learner is directly associated to three dynamic indicators collected from the activity done by the learner, namely the Number of Errors (NE), Number of Clicks (NC) and the time spent per activity $(\mathrm{T})$. We affected a weight to each indicator according to its significance in the completion of the activity. Therefore, the NE is assigned the highest weight since it is the most significant indicator followed by $\mathrm{NC}$ and finally the time per activity. Each user login to Codeclick using a username and a password and accesses his own session, which records the date and time of the access. Different forms of activities have been integrated (fill the gaps, puzzles, select list). Codeclick was inspired from the works of (Alammary et al., 2012), (Arnold and Pistilli, 2012) and (Murphy et al., 2009). The Figure 1 shows the architecture of Codeclick.

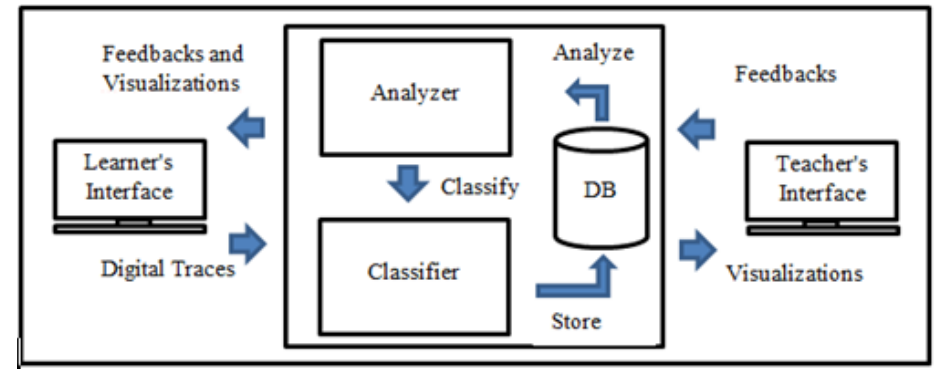

Figure 1. Codeclick architecture

\section{EXPERIMENT AND PRELIMINARY RESULTS}

In order to evaluate Codeclick, we conducted a preliminary experiment on two groups composed each by seven learners and for the same activity (the variables in Java). The learners were only boys with an average age of 19 years. The first group G1 used the F mode and the group G2 used the WF mode. Our goal was to assess the learners' performance level, to do this we relied on statistics based on the digital traces collected and recorded, more precisely indicators like the time spent per exercise, the number of clicks on the button validation (represent the number of errors), but also the number of clicks on the help button. These statistics are then used to generate personalized feedbacks and visualizations. Preliminary results of the experiment show that learners of the G1 achieved more satisfactory performance levels than those of the G2 in terms of errors made and time spent per exercise, thanks to the formative feedbacks and recommendations generated (see Fig. 2 and Fig.3). Furthermore, we noticed a correlation between the time spent per exercise, the number of errors made and the number of clicks on the help button. The Fig.4 depicts a feedback generated on error detection.
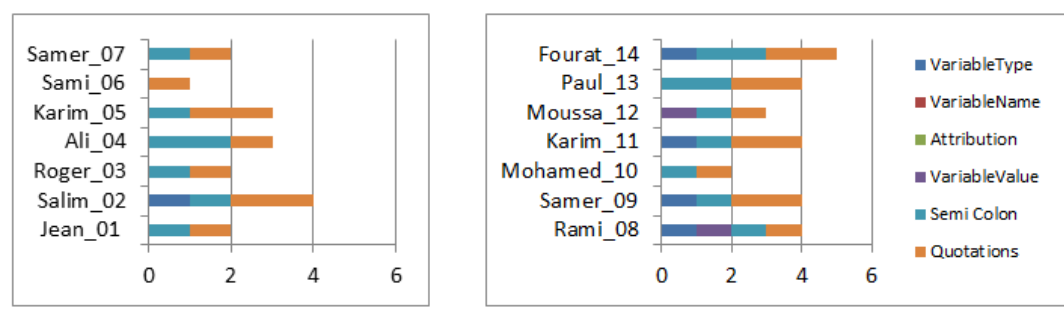

Figure 2. Visualizations representing the number and types of errors within groups G1 and $\mathrm{G} 2$ respectively in the activity the variables 

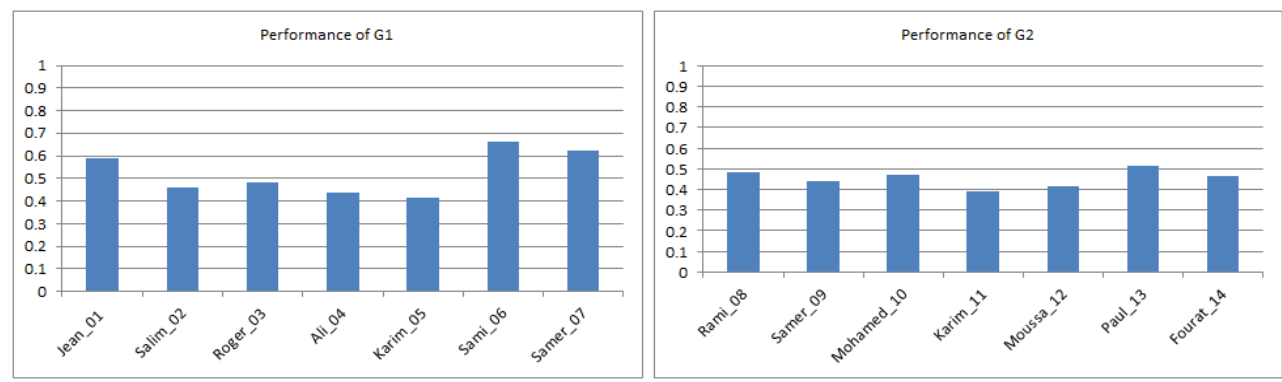

Figure 3. Visualizations representing the performance of G1 and G2 according to equation (1)

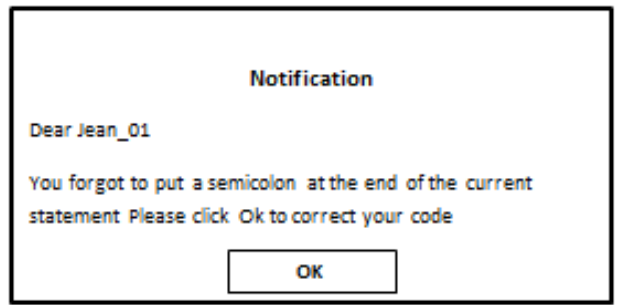

Figure 4. Example of feedback delivered on error detection

\section{CONCLUSION}

Practical learning of programming can be improved by performing appropriate activities, supported by a personalized feedback mechanism based on the learners' digital traces. In this article, we introduce a new programming learning environment called Codeclick, capable to analyze the learners' digital traces and provide personalized feedbacks and recommendations to support them. Codeclick uses a LA statistical approach based on indicators to assess the learners' performance level and provides feedbacks and visualizations to both learners and teachers. We have evaluated and compared the learners' performance levels within two groups that used Codeclick in both F and WF modes. Preliminary result shows that the learners within G1 privileged by feedbacks achieve more satisfactory performance levels in terms of number of errors and spend less time on exercises. Moreover they rarely use the help button. In future work we aim to validate the results on large scale groups and improve the analysis phase of Codeclick to support more complex programming activities while exploring other performance indicators.

\section{REFERENCES}

Alammary, A., Carbone, A., Sheard, J., 2012. Implementation of a Smart Lab for Teachers of Novice Programmers 123, 10.

Arnold, K.E., Pistilli, M.D., 2012. Course signals at Purdue: using learning analytics to increase student success, in: Proceedings of the 2nd International Conference on Learning Analytics and Knowledge - LAK '12. Presented at the the 2nd International Conference, ACM Press, Vancouver, British Columbia, Canada, p. 267.

Arnow, D., Barshay, O., 1999. WebToTeach: an interactive focused programming exercise system, in: FIE'99 Frontiers in Education. 29th Annual Frontiers in Education Conference. Designing the Future of Science and Engineering Education. Conference Proceedings

Bennedsen, J., Caspersen, M.E., 2007. Failure Rates in Introductory Programming. SIGCSE Bull. 39, 32-36.

Broisin, J., Venant, R., Vidal, P., 2017. Lab4CE: A Remote Laboratory for Computer Education. International Journal of Artificial Intelligence in Education 27, 154-180.

Corrin, L., de Barba, P., 2015. How Do Students Interpret Feedback Delivered via Dashboards?, in: Proceedings of the Fifth International Conference on Learning Analytics And Knowledge, LAK '15. ACM, New York, NY, USA, pp. 430-431. 
Ferguson, P., 2011. Student perceptions of quality feedback in teacher education. Assessment \& Evaluation in Higher Education 36, 51-62.

Gulwani, S., Radiček, I., Zuleger, F., 2014. Feedback Generation for Performance Problems in Introductory Programming Assignments, in: Proceedings of the 22Nd ACM SIGSOFT International Symposium on Foundations of Software Engineering, FSE 2014. ACM, New York, NY, USA, pp. 41-51.

Hattie, J., Timperley, H., 2007. The Power of Feedback. Review of Educational Research 77, 81-112.

Keuning, H., Jeuring, J., Heeren, B., 2019. A systematic literature review of automated feedback generation for programming exercises. ACM Transactions on Computing Education (TOCE) 19, 3.

Khan, I., Pardo, A., 2016. Data2U: scalable real time student feedback in active learning environments. pp. 249-253.

Lahtinen, E., Ala-Mutka, K., Järvinen, H.-M., 2005. A Study of the Difficulties of Novice Programmers, in: Proceedings of the 10th Annual SIGCSE Conference on Innovation and Technology in Computer Science Education, ITiCSE '05. ACM, New York, NY, USA, pp. 14-18.

Murphy, C., Kaiser, G., Loveland, K., Hasan, S., 2009. Retina: Helping students and instructors based on observed programming activities. Presented at the ACM Sigcse Bulletin, pp. 178-182.

Nunn, S., Avella, J.T., Kanai, T., Kebritchi, M., 2016. Learning Analytics Methods, Benefits, and Challenges in Higher Education: A Systematic Literature Review. OLJ 20.

Pardo, A., Bartimote-Aufflick, K., Buckingham Shum, S., Dawson, S., Gao, J., Gašević, D., Leichtweis, S., Liu, D., Martinez-Maldonaldo, R., Mirriahi, N., Moskal, A.C.M., Schulte, J., Siemens, G., Vigentini, L., 2018. OnTask: Delivering Data-Informed, Personalized Learning Support Actions. JLA 5, 235-249.

Pardo, A., Dawson, S., Dawson, S., 2015. Learning Analytics: How Can Data Be Used to Improve Learning Practice? [WWW Document]. Measuring and Visualizing Learning in the Information-Rich Classroom.

Siemens, G., Long, P., 2011. Penetrating the Fog: Analytics in Learning and Education. EDUCAUSE Review 5, 30-32.

Villamañe, M., Alvarez, A., Larrañaga, M., 2018. Supporting competence-based learning with visual learning analytics and recommendations, in: 2018 IEEE Global Engineering Education Conference (EDUCON). Presented at the 2018 IEEE Global Engineering Education Conference (EDUCON), pp. 1572-1575. 